\title{
Nucleosome Assembly Protein 1-Like 1 (Nap1I1) Regulates the Proliferation of Murine Induced Pluripotent Stem Cells
}

\author{
Yuan Yana,b Peipei Yin ${ }^{\mathrm{a}}$ Hui Gong ${ }^{\mathrm{a}}$ Yuanyuan Xue ${ }^{\mathrm{a}}$ Guoping Zhang ${ }^{\mathrm{a}}$ Bo Fang ${ }^{\mathrm{a}}$ \\ Zhidan Chen ${ }^{a}$ Yang Li $i^{a}$ Chunjie Yang ${ }^{a}$ Zheyong Huang ${ }^{a}$ Xiangdong Yang ${ }^{a}$ \\ Junbo Ge Yunzeng Zou $^{\text {a }}$
}

aShanghai Institute of Cardiovascular Diseases, Zhongshan Hospital and Institutes of Biomedical Sciences, Fudan University, Shanghai, China, bepartment of Histology and Embryology, College of Basic Medicine, Southern Medical University, Guangzhou, China

\section{Key Words}

Nap1l1 • iPS • Proliferation • Apoptosis • Cell cycle

\begin{abstract}
Background/Aims: To investigate whether nucleosome assembly protein 1-like 1 (Nap1l1) regulates the proliferation of induced pluripotent stem cells (iPSC) and the potential mechanisms. Methods: Nap1l1-knockdown-iPSC and Nap1l1-overexpression-iPSC were constructed by transfection of lentiviral particles. The proliferation of iPSC was detected by MTT analysis, and cell cycle was analyzed by flow cytometry. Results: Nap1l1 overexpression promoted iPSC proliferation and induced G2/M transition compared to their control iPSC while Nap1l1-knockdown-iPSC dramatically displayed the reduced proliferation and accumulated G2/M phase cells. Further analysis showed that Nap1l1 overexpression in iPSC increased the expression of cyclin B1, downregulated the expression of p21 and p27, while knockdown of Nap1l1 showed the opposite effects. In addition, overexpression of Nap1l1 promoted the phosphorylation of AKT and ERK in iPSC, while knockdown of Nap1l1 inhibited the effects. However, these effects displayed in Nap1l1-overexpression-iPSC were greatly suppressed by the inhibition of AKT or ERK signaling. Conclusions: The results indicate that Nap1l1 promotes the proliferation of iPSC attributable to G2/M transition caused by downregulation of p27 and p21, and upregulation of cyclin B1, the activation of AKT or ERK is involved in the process. The present study has revealed a novel molecular mechanism involved in the proliferation of iPSC.
\end{abstract}

(C) 2016 The Author(s)

Published by S. Karger AG, Basel

\section{Introduction}

The generation of induced pluripotent stem cells (iPSC) by the forced expression of four special transcription factors (Oct3/4, Sox2, Klf4, and c-Myc) into somatic cells, provides new

Y. Yan and P. Yin contributed equally to this work. 
prospects for disease-related basic research and regenerative medicine [1-3]. These cells and embryonic stem cells (ESC) share many similarities, including self-renew and multilineage differentiation [4-8]. Cell-based therapy with healthy iPSC-derived cells holds great promise for repair of diseased tissue. As compared to ESC, iPSC show more advantages such as avoiding ethical concerns and immune rejection problems of cell-based transplantation $[1,5]$. With the development of iPSC research, patient-specific iPSC not only provide an autologous cell source, but also become disease models in vitro for drug screening or understanding of molecular mechanisms [9]. However, iPSC technology still faces some challenges such as high variability and low efficiency. It is a time-consuming process to get enough number of patient-specific iPSC for therapeutic applications. So how to enhance the rapid proliferation of iPSC are of great interest.

Nucleosome assembly protein-1 (Nap1) and the related Nap1 family have been reported to be involved in nucleosome assembly as well as cell cycle progression or cell proliferation $[10,11]$. Nap1 overexpression also lead to increase in endothelial growth [10]. In Drosophila melanogaster, Nap1 deletion causes early embryonic lethality, although the underlying mechanism has not been determined [12]. Nap1-like 1(Nap1l1), one of Nap1-like proteins, has some similar activities to Nap1 such as nucleosome assembling, however, it shows higher nucleosome disassembly activity [11]. Previous reports have shown that Nap111 and Nap1l2 are required for root growth in Arabidoposis [13]. Nap1l1 mRNA and protein level in T-cells both are rapidly increased in response to mitogenic stimulation [14]. Recently, we observed that Nap111 promoted mesodermal induction and cardiomyocytes differentiation [15]. Some studies have identified that Nap1l1 is highly expressed in some tumor tissues such as hepatoblastomas [16] and small intestinal carcinoid [17]. Cancer cells and iPSC are known to share some common characteristics with regard to self-renew, rapid proliferation and indefinite growth. Therefore, Nap1l1 might also be involved in the regulatory network for proliferation of iPSC.

To address this issue, we constructed Nap1l1-knockdown-iPSC and Nap1l1overexpression-iPSC to examine its effect on the proliferation of iPSC. The present study will be helpful to clarify a novel mechanism for the proliferation of iPSC.

\section{Materials and Methods}

\section{iPSC Culture}

The murine iPS clone iPS-MEF-Ng-20D-17 (APS0001), reprogrammed by the transduction with retroviral vectors encoding the 4 transcription factors Oct 4, Sox 2, c-Myc, and Klf 4, was obtained from RIKEN BioResource Center, Japan. Cells were grown on mitotically inactivated murine embryonic fibroblasts (MEFs) in 6-well culture plates and cultured in Knockout Dulbecco's modified Eagle's medium (DMEM) (Invitrogen, Carlsbad, CA, USA), supplemented with 20\% Knockout serum replacement (Invitrogen, Carlsbad, CA, USA), $1 \%$ nonessential amino acids (Invitrogen, Carlsbad, CA, USA), $0.1 \mathrm{mM} \beta$-mercaptoethanol (Sigma-Aldrich, St. Louis, MO, USA), $0.2 \mathrm{mmol} / \mathrm{L}$ L-glutamine (Invitrogen, Carlsbad, CA, USA) and 1000 units/ml murine leukemia inhibitory factor.

\section{Generation of Nap1l1-knockdown-iPSC and Nap1l1-overexpression-iPSC}

Generation of Nap1l1-knockdown-iPSC and Nap1l1-overexpression-iPSC was described in our previous study [11]. In brief, mouse Nap1l1 short-hairpin RNA (shRNA) construct was developed by subcloning the sequence (5'-CCG GCG ATC CAG ACT ATG ACC CAA ACT CGA GTT TGG GTC ATA GTC TGG ATC GTT TTTG-3') into the MISSION-shRNA vector (derived from pLKO.1-puro; Thermo Fisher Scientific Inc, Waltham, MA, USA). The pLOC vector encoding the full length of the Nap1l1 gene (NCBI reference sequence ID, NM_001146707.1) was constructed by Thermo Fisher Scientific Inc (Waltham, MA, USA). A lentiviral shRNA-sramble-pLKO.1 vector and pLOC vector were used as a control, respectively. Lentiviral particles were generated and transfected into iPSC, and these cells were selected with $2 \mu \mathrm{g} / \mathrm{ml}$ of puromycin for one week.

\section{KARGER}




\section{Cellular Physiology Cell Physiol Biochem 2016;38:340-350 \begin{tabular}{l|l|l} 
DOI: 10.1159/000438634 2016 & $\begin{array}{l}\text { () 2016 The Author(s). Published by S. Karger AG, Basel } \\
\text { www.karger.com/cpb }\end{array}$
\end{tabular} \\ Yan et al.: The Effect of Nap1l1 on the Proliferation of iPSC}

Quantitative Real-Time Polymerase Chain Reaction ( $q R T-P C R$ )

Total RNA was extracted from iPSC using Trizol reagent (Invitrogen, Carlsbad, CA) and convert into cDNA as described previously [15]. Quantitative Real-Time PCR was performed with Power SYBR Green PCR Master Mix (TaKaRa, Shiga, Japan) in the iCycler System (Bio-Rad, Philadelphia, PA, USA). Relative levels of gene expression were normalized to the glyceraldehyde-3-phosphate dehydrogenase (GAPDH) gene by using the comparative Ct method according to the manufacturer's instructions.

\section{Western blot analysis}

Cells and tissues were lysed in radioimmunoprecipitation (RIPA) buffer. Protein concentration was determined by bicinchoninic acid (BCA) assay (Thermo Fisher Scientific Inc, Rockford, IL USA). The proteins were resolved in 12\% SDS polyacrylamide gels and transferred onto nitrocellulose membrane. The membranes were then incubated overnight at $4{ }^{\circ} \mathrm{C}$ in blocking solution containing $5 \%$ nonfat dry milk in PBS with $0.1 \%$ Tween-20. Subsequently the membranes were incubated with primary antibody against Nap1l1 (Abcam, Cambridge, MA, USA), extracellular signal-regulated kinase (ERK), p-ERK, p-AKT, AKT (Cell Signaling, Danvers, MA, USA), or GAPDH (Kangcheng, Shanghai, China) followed by incubation with HRP (horseradish peroxidase)-conjugated second antibodies (DINGGUO Biotechnology, Beijing). Detection of HRP was performed by Las3000 (FUJIFILM Inc., Tokyo, Japan).

\section{Cell viability assay}

Cell viability was assessed using the 3-(4, 5-dimethyl-2-thiazolyl)-2,5-diphenyl-2-H-tetrazolium bromide (MTT) assay as previous study [18]. Cell lines were plated at a density of $5 \times 10^{3}$ cells per well on 96-well plates in $100 \mu \mathrm{l}$ of culture medium. At the indicated time, MTT labeling mixture $(10 \mu \mathrm{L}, 5 \mathrm{mg} / \mathrm{mL})$ was added to each well and incubated for an additional $4 \mathrm{~h}$. The formazan precipitate was dissolved in 150 $\mu \mathrm{L}$ dimethyl sulfoxide and measured with a DTX-880 Multimode Detector (Beckman Coulter, USA) at 570 nm. Assays were performed in triplicate.

\section{Alkaline phosphatase staining}

Cells were seeded at a density of 2000 per 6 -cm dishes. After 7 days, the colonies were fixed with $50 \%$ acetone and $50 \%$ methanol at room temperature for 2 minutes and stained using an alkaline phosphatase (AP) staining kit (Vector Laboratories, Burlingame, CA) according to a standard protocol.

\section{Flow cytometric analysis}

For cell-cycle analysis, cells $\left(1 \times 10^{6}\right.$ cells in $1 \mathrm{~mL}$ of medium $)$ were fixed with $70 \%$ ethanol at $4^{\circ} \mathrm{C}$ for at least $12 \mathrm{~h}$ as previous study [19]. The cells were incubated in RNase A $(50 \mu \mathrm{g} / \mathrm{mL})$ at $37^{\circ} \mathrm{C}$ for 30 min. Intracellular DNA was labeled with propidium iodiode (PI) $(50 \mu \mathrm{g} / \mathrm{mL})$ and the cell-cycle profile was analyzed with a fluorescence-activated cell sorter (FACS) using Expo32 software (Expo32 Multi COMP and V1.2 Analysis).

\section{Immuofluorescence staining}

Cells were fixed using 4\% paraformaldhyde, permeated using 0.25\% Triton X-100 and blocked with $10 \%$ goat serum (Gibco, Carlsbad, CA USA). The cells were incubated overnight at $4{ }^{\circ} \mathrm{C}$ with rabbit polyclonal IgG anti-Nap1l1 antibody (1:200, Proteintech, Chicago, IL, USA), then rinsed 3 times for 10 min. Further incubation was performed with the FITC-conjugated anti-rabbit secondary antibody (1:1000, Invitrogen, Carlsbad, CA) for $1 \mathrm{~h}$ at room temperature. The cells were rinsed once more, counterstained with 4', 6'-diamidino-2-phenylindole (DAPI) (Sigma-Aldrich, St. Louis, MO, USA), and analyzed with Axio Observer A1 fluorescence microscope (Zeiss, Munich, Germany).

\section{Statistical analysis}

All data were showed as mean \pm SEM. Any differences between Nap1l1-knockdown-iPSC or Nap1l1overexpression-iPSC and their control-iPSC were analyzed using an unpaired t-test as previous study [20]. Differences in the Nap1l1-overexpression-iPSC and control-iPSC with or without U0126 or MK2206 treatment were determined using a two-way analysis of variance. All the experiments were repeated at least three times. 


\section{Results}

Nap111 overexpression promotes the proliferation of iPSC

Our recent study revealed that Nap1l1 is expressed at high level in murine iPSC and ESC [15]. Nap1l1 is localized predominantly in the cytoplasm but sporadically in nucleus of iPSC [15]. To explore the role of endogenous Nap1l1 in the proliferation of iPSC, we established

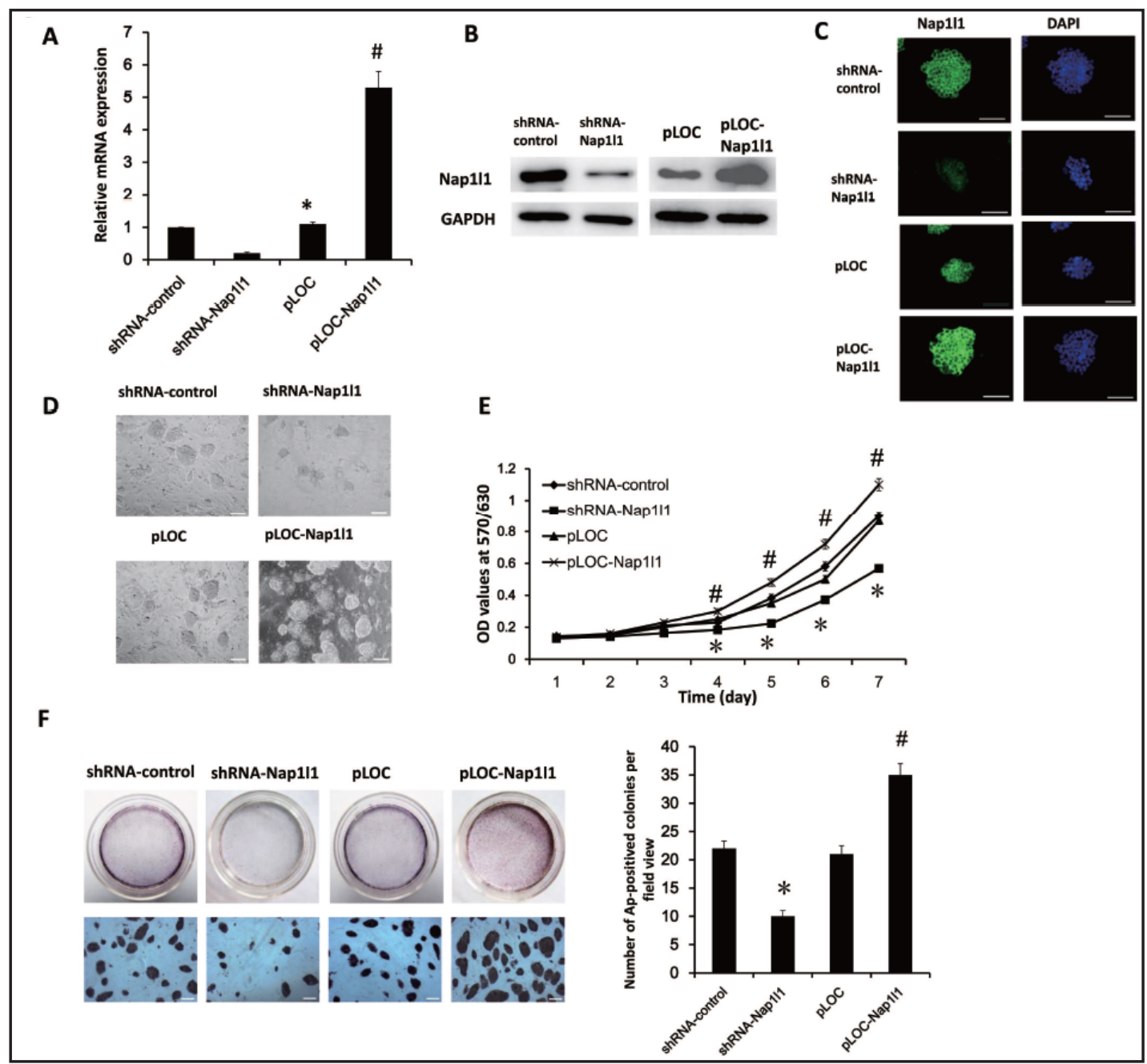

Fig. 1. Nap111 promotes the proliferation of iPSC. Nap111 mRNA and protein expressions were detected by qRT-PCR (A) and Western blot analysis (B) in Nap1l1- knockdown-iPSC, Nap111-overexpression-iPSC and their control iPSC. GAPDH expression was used as a loading control. Nap1l1 mRNA expression was showed as folds of value obtained in shRNA-control-iPSC. (C) Immunostaining of Nap1l1 in Nap1l1-knockdown-iPSC, Nap111-overexpression-iPSC and control iPSC with antibodies to Nap111 (green). Nuclei were stained with DAPI (blue). (D) Morphology of Nap111-knockdown-iPSC and Nap1l1-overexpression-iPSC was observed under optical microscope after cultured 5 days. (E) The growth rate of Nap111-knockdown-iPSC and Nap111-overexpression-iPSC was measured by MTT assay. (F) The colony number from Nap111-knockdown-iPSC, Nap1l1-overexpression-iPSC and their control iPSC was quantified by AP staining. Left upper panel: photographs of the colonies. Left lower panel: high-magnification images of AP-staining. The graph showed the AP-positive numbers per field of view $(n=3 /$ well). Murine iPSC were dissociated and then plated on a feeder cell layer at a clonal density for 5 days in the presence of LIF. Then, the colonies were stained with AP (dark purple). AP: alkaline phosphatase. Nap111- knockdown-iPSC: shRNA-Nap111, Nap1l1-overexpression-iPSC: pLOC-Nap1l1. Control iPSC: shRNA-control or pLOC. Scale bars $=200 \mu \mathrm{m}$. Values are means \pm S.E.M; ${ }^{*}$ p $<0.05$ (shRNA-Nap111 vs. shRNA-control); \# p < 0.05 (Nap1l1 vs. pLOC). Any experiment was repeated independently at least 3 times.

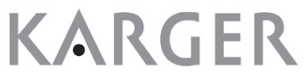




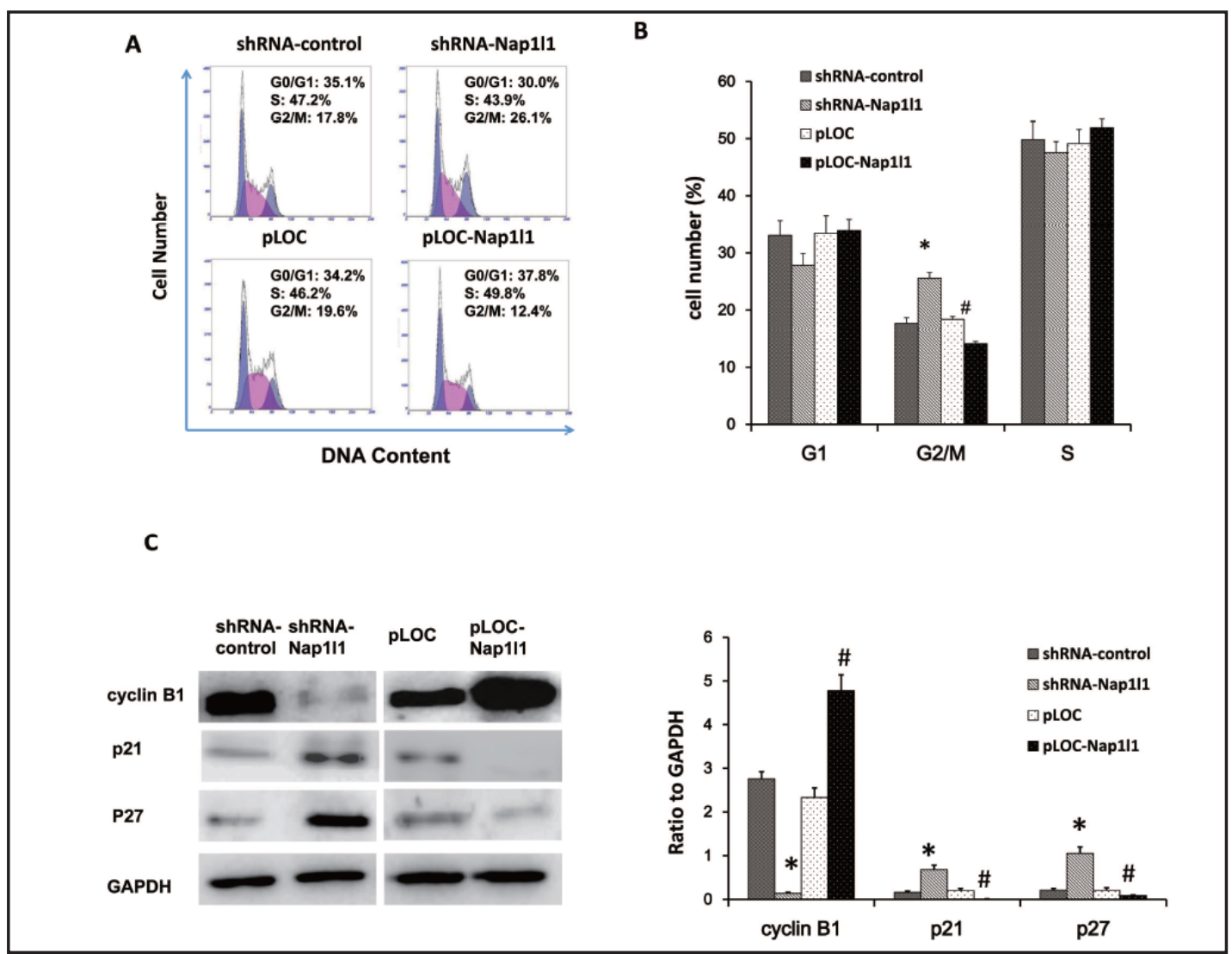

Fig. 2. Effect of Nap1l1 on the progression of iPSC cycle. (A) Cell cycle distributions were monitored by flow cytometric analysis with PI staining in Nap1l1-knockdown-iPSC, Nap1l1-overexpression-iPSC and control iPSC. (B) The graph displayed the percentage of G1 phase, G2/M phase and S phase of iPSC. (C) Cell cycle regulatory proteins, cyclin B1, p21 and p27, were detected by western blot analysis in iPSC. GAPDH was used as an internal control. Nap1l1- knockdown-iPSC: shRNA-Nap1l1, Nap1l1-overexpression-iPSC: pLOC-Nap111. Control iPSC: shRNA-control or pLOC. Each column was normalized by GAPDH. Values are means \pm S.E.M; ${ }^{*}$ p $<0.05$ (shRNA-Nap1l1 vs shRNA-control); \# p < 0.05 (Nap1l1 vs pLOC ). Any experiment was repeated independently at least 3 times.

Nap1l1-knockdown- or overexpression-iPSC as our recent study [15]. The shRNA-scramble vector or empty lentiviral vector was transfected into iPSC as control, respectively. Realtime RT-PCR and western blot analysis showed that the levels of Nap1l1 mRNA and protein were dramatically decreased in Nap1l1-knockdown-iPSC, while the expressions were significantly increased in Nap1l1-overexpression-iPSC compared with their control cells, respectively (Fig. 1A, 1B).

To evaluate the effect of Nap1l1 on proliferation in vitro, iPSC were cultivated in the presence of LIF and MEF. After cultured for 5 days, Nap1l1-knockdown- and Nap1l1overexpression-iPSC both showed typical oval-shaped compact colonies (Fig. 1C, 1D). Knockdown of Nap1l1 reduced, while, overexpression of Nap1l1 increased, the number and size of colonies from iPSC compared with control iPSC, respectively, suggesting that Nap1l1 might be involved in the control of cell proliferation in iPSC. We then performed MTT assay to measure the growth rate of iPSC. Nap1l1-knockdown-iPSC showed a decrease in growth rate and Nap1l1-overexpression-iPSC displayed an increase in growth rate compared to their control cells, respectively (Fig. 1D,1E).

We then performed a colony forming assay to examine the effect of Nap111 on selfrenewal ability of iPSC. iPSC were seeded at low density (1000 or 2000 cells per 35-mm dish) in the presence of LIF and cultured for 7 days to form cell colonies. We evaluated 


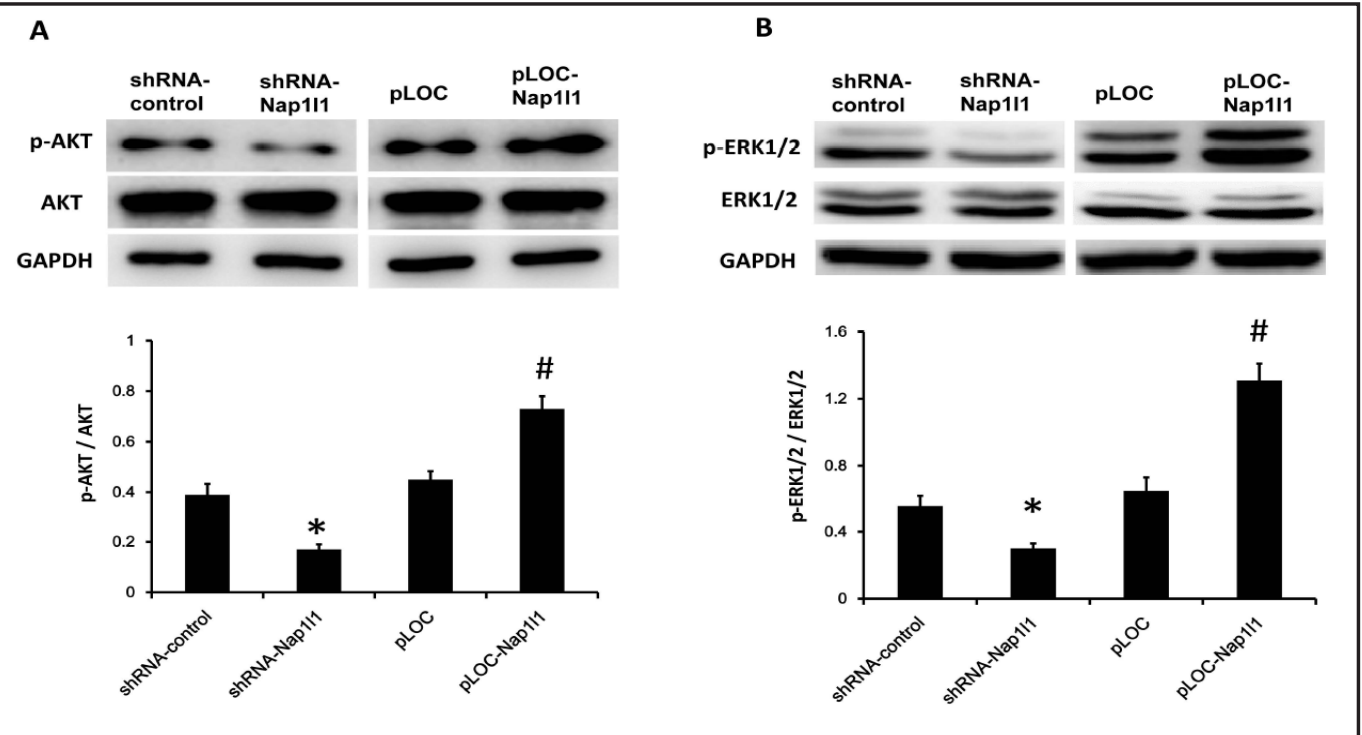

Fig. 3. Nap1l1 is involved in AKT and ERK signaling pathways. (A) Expressions of p-AKT and AKT were analyzed by western blot analysis in iPSC. The graph displayed the ratio of p-AKT to total AKT. (B) Expressions of p-ERK1/2 and ERK1/2 were analyzed by western blot analysis in iPSC. The graph showed the ratio of p-ERK to total ERK. GAPDH was detected as internal control. Nap1l1- knockdown-iPSC: shRNA-Nap1l1, Nap1l1-overexpression-iPSC: pLOC-Nap1l1. Control iPSC : shRNA-control or pLOC. Each column was normalized by GAPDH. Values are means \pm S.E.M; *p < 0.05 (shRNA-Nap1l1 vs shRNA-control); \# p < 0.05 (Nap1l1 vs control ). Any experiment was repeated independently at least 3 times.

colony morphology and AP (alkaline phosphatase) activity in iPSC cultured in the presence of LIF. The AP-staining, which is indicative of the undifferentiated property of ESC or iPSC, was not affected by knockdown or overexpression of Nap1l1 in iPSC. However, the number of colonies declined drastically in Nap1l1-knockdown-iPSC but greatly increased in Nap1l1overexpression-iPSC in comparison with their control iPSC (Fig. 1F).

Taken together, our results indicate Nap1l1 promotes the proliferation of iPSC.

Nap1l1 regulates the progression of the iPSC cycle

It has been reported that Nap1 exerts biological effects on cell cycle progression [21-24]. To test whether Nap1l1 is involved in the progression of the iPSC cycle, flow cytometry was used to analyze the cells cycle profile of Nap1l1 knockdown- and Nap1l1-overexpressioniPSC. The control-iPSC showed normal cell cycle profile. Nap1l1 knockdown in iPSC induced a higher number of cells in $\mathrm{G} 2 / \mathrm{M}$ phase while Nap1l1 overexpression resulted in fewer cells in $\mathrm{G} 2 / \mathrm{M}$ phase (Fig. 2A, 2B). Thus, it suggests that Nap1l1 overexpression enhances the proliferation of iPSC by promoting cell cycle progression in G1 phase.

To further understand how Nap1l1 regulates the cell cycle progression, we performed western blot analysis of iPSC for cyclin B1 (G2/M phase-specific cyclin), p21 and p27 levels (inhibitors of cyclin-dependent kinase). Knockdown of Nap1l1 in iPSC reduced the level of cyclin B1 and upregulated the levels of p21 and p27, while overexpression of Nap1l1 increased the level of cyclin B1 and decreased the expression of p21 and p27 in iPSC (Fig. 2C).

These results reveal that Nap1l1 promotes G2/M transition and subsequently enhances the proliferation of iPSC by regulating the expression of cyclin B1, p21 and p27.

Nap1l1 induces the phosphorylation of AKT and ERK in iPSC

PI3K/AKT and MEK/ERK signals have been shown to play key roles in proliferation and apoptosis of cells $[25,26]$. To investigate the underlying pathways which are involved in the regulation of Nap111 on iPSC proliferation, we detected the levels of phosphorylation of AKT 
Fig. 4. The effect of MEK or AKT inhibitor on the proliferation of Nap1l1-overexpression-iPSC. Morphology of iPSC was observed in optical microscope. (B) The growth rate of iPSC was measured by MTT assay. Control-iPSC and Nap1l1-overexpression-iPSC were treated with or without MK2206 or U0126, DMSO was used as control. Nap1l1-overexpression-iPSC: pLOC-Nap111. Control iPSC: pLOC. Scale bars $=200 \mu \mathrm{m} .{ }^{*} \mathrm{p}<$ 0.05 vs DMSO+pLOC group. \# $\mathrm{p}<0.05$ vs DMSO+Nap1l1-pLOC group. Any experiment was repeated independently at least 3 times.

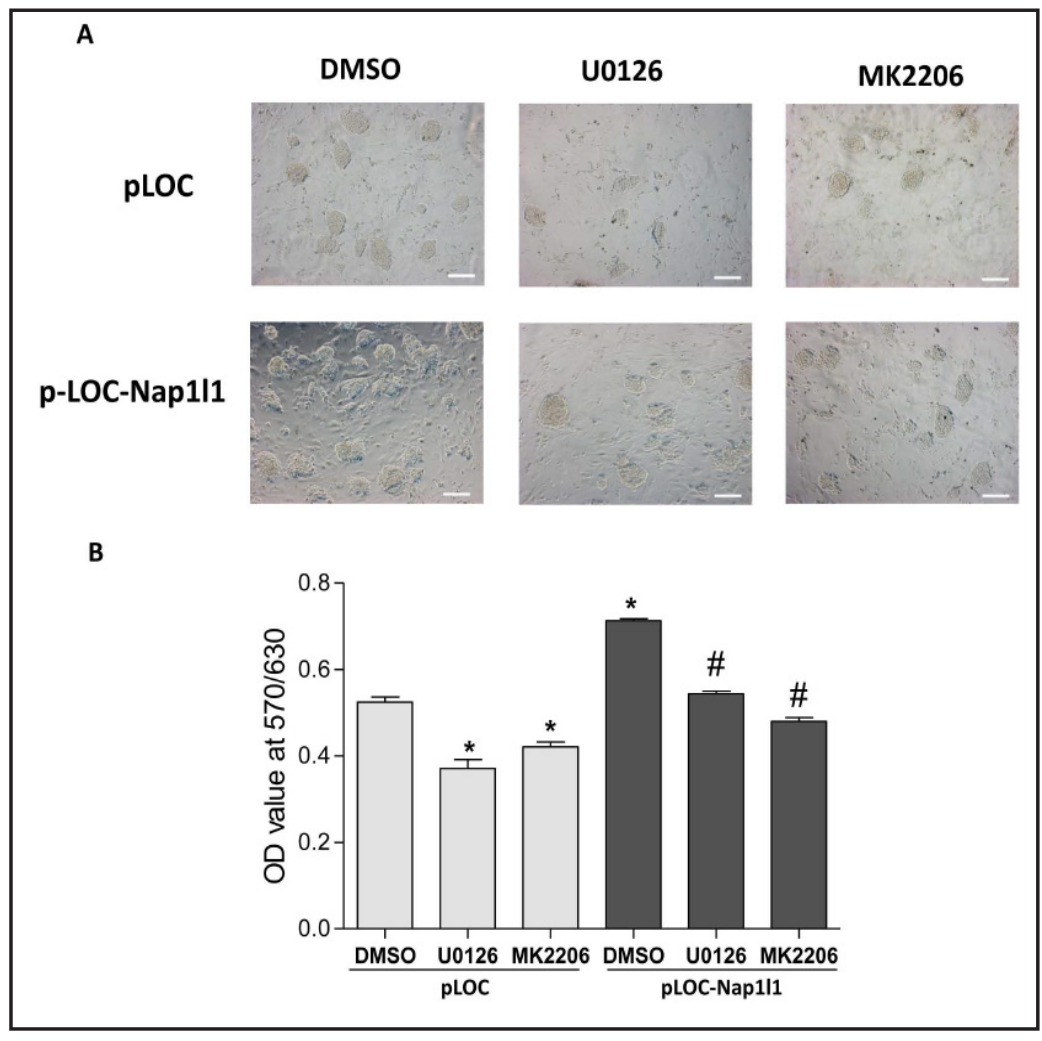

and ERK in iPSC. Nap1l1 knockdown significantly decreased phosphorylation levels of AKT and ERK, whereas Nap1l1 overexpression showed opposite effects in iPSC (Fig. 3A, 3B).

Therefore, our results suggest that Nap1l1 possibly modulates proliferation of iPSC through AKT and ERK pathways.

Inhibition of AKT or ERK activation greatly reverses the proliferous effect of iPSC induced by Nap1l1 overexpression

To explore whether Nap1l1 regulates the proliferation of iPSC by ERK or AKT pathway, we inhibited the activation of ERK or AKT in Nap1l1-overexpression-iPSC or control-iPSC to detect the proliferous effect. Here, we used the U0126, a specific inhibitor of MEK, which can efficiently inhibit the MEK-mediated phosphorylation of ERK [27], to inhibit ERK activation as previous study [28], while MK2206 was used as the AKT inhibitor in the present study. The results showed that U0126 or MK2206 greatly reversed the proliferous effect induced by Nap1l1 overexpression in iPSC. They both showed the inhibitory effect on the proliferation in control iPSC (Fig. 4A, 4B).

We then explored the expression of cyclin B1, p21 and p27 in iPSC with or without inhibition of AKT or ERK activation. In control-iPSC, U0126 or MK2206 increased the expression of p21 and p27 while they both decreased the expression of cyclin B1. The upregulation of cyclin B1, downregulation of p21 and p27 induced by Nap1l1-overexpression in iPSC were partly abolished by MK2206 or U0126 treatment (Fig. 5A, 5B). These data suggest that Nap1l1 overexpression regulates cell cycle proteins by activation of ERK or AKT to promote the proliferation of iPSC.

\section{Discussion}

Here, we present evidence demonstrating that murine iPSC express Nap1l1 and Nap1l1 is critical for the rapid proliferation of murine iPSC under non-stressful conditions. The downregulation of Nap1l1 leads to reduction of iPSC proliferation, which is probably due to G2/M phase arrest, while overexpression of Nap1l1 has shown opposite results. These 

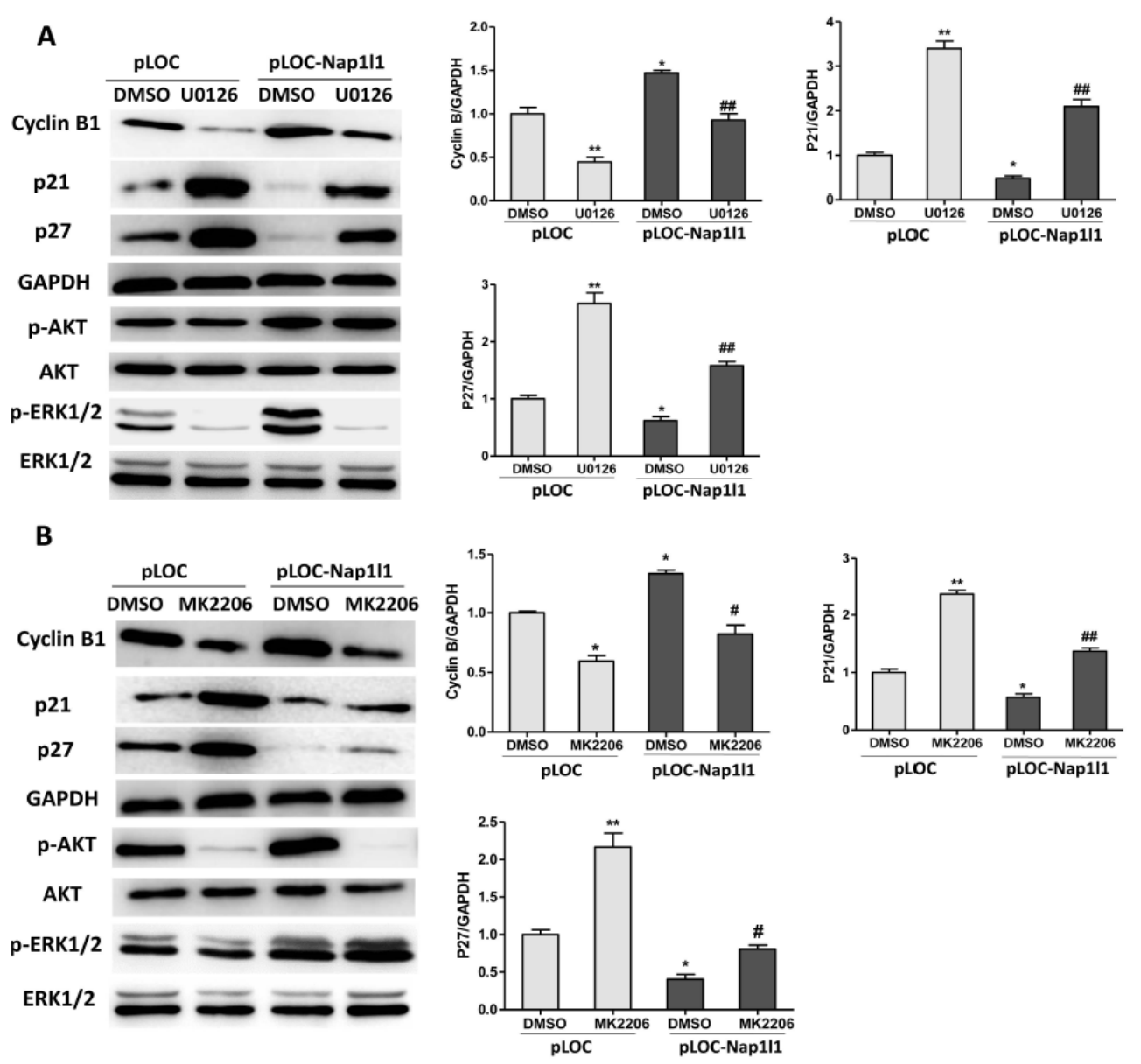

Fig. 5. The effect of MEK or AKT inhibitor on expression of the cell cycle regulatory proteins in Nap1l1-overexpression-iPSC. (A) Western blot analysis of cyclin B1, p21, p27, p-ERK, ERK, p-AKT and AKT in Nap1l1-overexpression-iPSC and control-iPSC treated with U0126 or DMSO. (B) Western blot analysis of cyclin B1, p21, p27, p-ERK, ERK, p-AKT and AKT in Nap1l1-overexpression-iPSC and control-iPSC treated with ME2206 or DMSO. GAPDH served as an internal control. Graphs showed these proteins level expressed as ratio of value from control iPSC treated with DMSO. *p $<0.05$; ${ }^{* *} \mathrm{p}<0.01$ vs DMSO+pLOC group. \# $\mathrm{p}<0.05$; \#\# p $<0.01$ vs DMSO+Nap1l1-pLOC group. Any experiment was repeated independently at least 3 times.

findings strongly support that cytoplasmic expression of Nap1l1 in iPSC is critical for rapid proliferation of iPSC.

Nap1, as conserved histone chaperone protein, is involved in the dynamical regulation of the histone H2A-H2B dimer. It has been reported to regulate DNA replication and chromatin formation which contribute to multiple process including the growth of cells [14, 21, 29]. Nap1l1, highly homologous to Nap1, plays overlapping roles in regulation of nucleosome assembly [30] and DNA replication [31]. It suggests Nap1l1 plays an important role in the process of cell proliferation. Overexpression of Nap1l1 has been reported to promote the proliferation of neuroendocrine neoplasms [32]. Our recent study revealed that Nap1l1 was expressed in iPSC at high level [15]. Whether Nap1l1 regulates the proliferation of stem cells especially iPSC hasn't been reported to date. The present study showed that knockdown of Nap1l1 reduced proliferation rate of iPSC, while overexpression of Nap1l1 increased their growth potential. These findings directly indicate that Nap1l1 is involved in the regulation of iPSC proliferation.

We next examined the cell cycle profile in Nap1l1-knockdown-iPSC or Nap1l1overexpression-iPSC. Cell cycle analysis using PI revealed knockdown of Nap1l1 in iPSC 
delayed the cell cycle in G2/M phase, whereas its overexpression promoted G2/M transition. It is well known that the major regulator of the G2 to M transition is the MPF (M-phase promoting factor), which comprises the catalytic subunit $\mathrm{Cdc} 2$ and the regulatory subunit cyclin B1 [33]. Cyclin B1, an important G2/M checkpoint regulator, usually induces cells from G2 to M phase [34]. The present study showed that Nap1l1 positively regulated cyclin B1 expression in iPSC. It suggests that overexpression of Nap1l1 induced G2/M transition by the upregulation of cyclin B1 in iPSC. Nap1 in the Xenopus embryo has been observed to interact with cyclin B1 [21], but the function is unclear. Whether Nap1l1 could interact with cyclin B1 to promote iPSC proliferation needs further study to elucidate.

P21 or p27, cyclin/cyclin-dependent kinase (CDK) inhibitor, has been reported to cause cell-cycle arrest and inhibit the proliferation of mammalian cells [35, 36]. It is known that p21 or p27 binds to cyclin, CDK subunits or cyclins-CDK complexes to inhibit their activities which are involved in cell cycle progression including G2/M arrest [35-37]. The expression of p21 or p27 has showed a negative correlation to the levels of some cyclins or CDKs in cancer cells [35, 38]. The present results showed that knockdown of Nap1l1 led to a significant increase in p21 and p27 expression while Nap1l1 overexpression resulted in the opposite effects in iPSC. These data imply that Nap1l1 inhibits the expression of p21 or p27 and subsequently promotes the activation of CDKs or the expression of cyclins to induce G2/M transition which results in the proliferation of iPSC.

P21 or p27 has been reported to be degraded by ubiquitination through interaction with Skp2, an E3 ubiquitin ligases. Skp2 recognizes Thr187-phosphorylated p27 to promote poly-ubiquitination and subsequent degradation [35]. Whether Nap1l1 increases phosphorylation of p21 or p27 and enhances Skp2-p21 or Skp2-p27 association in iPSC needs further investigation. Huang et al. reported that activation of p53 and p21 was related to the inactivation of PI3K/AKT and ERK signaling pathways during virus replication [39]. PI3K/ AKT or MEK/ERK pathways, has been reported to control proliferation and differentiation of stem cells [26-28]. The present study suggests that Nap1l1 activates ERK or AKT pathway to promote the proliferation by downregulation of p21 and p27 and upregulation of cyclin B1 in iPSC. Su CC reported that Tanshinone IIA inhibits the proliferation of human gastric cancer cells by G2/M phase arrest which results from inhibition of ERK [26]. Chang $\mathrm{CH}$ reported that hydronephrotic urine promotes urothelial carcinoma cell proliferation or migration through the activation of AKT and ERK signaling pathway to decrease the expression of p21 and p27 [40]. These results are similar to our study. It suggests that Nap1l1 activates AKT or ERK, the activation of AKT or ERK signaling induces the degradation of p21 or p27 which results in G2/M transition and the proliferation of iPSC. Schimmack et al. reported that Nap1l1 enhanced p57 (another CDK inhibitor) promoter methylation and subsequently induced the protein degradation to promote the pancreatic neuroendocrine neoplasm proliferation [32]. Whether Nap1l1 regulates the proliferation of iPSC by the methylation of p57 is unclear. In addition, there is a dynamic equilibrium between cellular proliferation and apoptosis under physiological conditions. Inhibition of cellular apoptosis can cause induction of cell growth. However, few studies report the effect of Nap1l1 on cellular apoptosis. Whether Nap1l1 overexpression promotes the proliferation of iPSC partly results from the inhibition of apoptosis needs further study to clarify. The limitation of this study is that we didn't explore how Nap1l1 regulates the activation of ERK or AKT or whether Nap1l1 promotes the proliferation of iPSC by inhibition of cellular apoptosis.

In summary, Nap1l1 activates AKT or ERK signaling, then downregulates the expression of p21 and p27 and subsequently upregulates the expression of cyclin B1 which contribute to the proliferation of iPSC. The present study has revealed a novel molecular mechanism involved in the proliferation of iPSC.

\section{Acknowledgments}

This work was supported by Technology Commission of Shanghai Municipality (13JC1401703) and National Natural Science Fund of China (81370258; 81370003). 


\section{Cellular Physiology Cell Physiol Biochem 2016;38:340-350

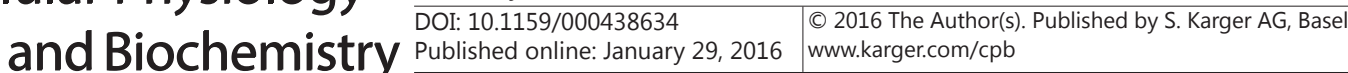

Yan et al.: The Effect of Nap1l1 on the Proliferation of iPSC

\section{Disclosure Statement}

The authors indicate no potential conflicts of interest.

\section{References}

1 Takahashi K, Yamanaka S: Induction of pluripotent stem cells from mouse embryonic and adult fibroblast cultures by defined factors. Cell 2006;126:663-676.

2 Mali P, Ye Z, Hommond HH, Yu X, Lin J, Chen G, Zou J, Cheng L: Improved efficiency and pace of generating induced pluripotent stem cells from human adult and fetal fibroblasts. Stem Cells 2008;26:1998-2005.

3 Vallier L, Touboul T, Brown S, Cho C, Bilican B, Alexander M, Cedervall J, Chandran S, Ahrlund-Richter L, Weber A, Pedersen RA: Signaling pathways controlling pluripotency and early cell fate decisions of human induced pluripotent stem cells. Stem Cells 2009;27:2655-2666.

4 Hanna J, Cheng AW, Saha K, Kim J, Lengner CJ, Soldner F, Cassady JP, Muffat J, Carey BW, Jaenisch R: Human embryonic stem cells with biological and epigenetic characteristics similar to those of mouse escs. P Natl Acad Sci USA 2010;107:9222-9227.

5 Chin MH, Pellegrini M, Plath K, Lowry WE: Molecular analyses of human induced pluripotent stem cells and embryonic stem cells. Cell Stem Cell 2010;7:263-269.

6 Xu R, Srinivasan SP, Sureshkumar P, Nembo EN, Schafer C, Semmler J, Matzkies M, Albrechtsen M, Hescheler J, Nguemo F: Effects of synthetic neural adhesion molecule mimetic peptides and related proteins on the cardiomyogenic differentiation of mouse embryonic stem cells. Cell Physiol Biochem 2015;35:2437-2450.

7 Ivanyuk D, Budash G, Zheng Y, Gaspar JA, Chaudhari U, Fatima A, Bahmanpour S, Grin VK, Popandopulo AG, Sachinidis A, Hescheler J, Saric T: Ascorbic acid-induced cardiac differentiation of murine pluripotent stem cells: Transcriptional profiling and effect of a small molecule synergist of wnt/beta-catenin signaling pathway. Cell Physiol Biochem 2015;36:810-830.

8 Cho GS, Fernandez L, Kwon C: Regenerative medicine for the heart: Perspectives on stem-cell therapy. Antioxid Redox Signaling 2014;21:2018-2031.

9 D'Aiuto L, Zhi Y, Kumar Das D, Wilcox MR, Johnson JW, McClain L, MacDonald ML, Di Maio R, Schurdak ME, Piazza P, Viggiano L, Sweet R, Kinchington PR, Bhattacharjee AG, Yolken R, Nimgaonka VL: Large-scale generation of human ipsc-derived neural stem cells/early neural progenitor cells and their neuronal differentiation. Organogenesis 2014;10:365-377.

10 Clark J, Alvarez DF, Alexeyev M, King JAC, Huang L, Yoder MC, Stevens T: Regulatory role for nucleosome assembly protein-1 in the proliferative and vasculogenic phenotype of pulmonary endothelium. Am J Physiol Lung Cell Mol Physiol 2008;294:L431-L439.

11 Okuwaki M, Kato K, Nagata K: Functional characterization of human nucleosome assembly protein 1-like proteins as histone chaperones. Genes Cells 2010;15:13-27.

12 Lankenau S, Barnickel T, Marhold J, Lyko F, Mechler BM, Lankenau DH: Knock-out targeting of the drosophila nap1 gene and examination of DNA repair tracts in the recombination products. Genetics 2003;163:611-623.

13 Zhu Y, Dong AW, Meyer D, Pichon O, Renou JP, Cao KM, Shen WH: Arabidopsis nrp1 and nrp2 encode histone chaperones and are required for maintaining postembryonic root growth. Plant Cell 2006;18:2879-2892.

14 Simon HU, Mills GB, Kozlowski M, Hogg D, Branch D, Ishimi Y, Siminovitch KA: Molecular characterization of hnrp, a cdna-encoding a human nucleosome-assembly-protein-i-related gene-product involved in the induction of cell-proliferation. Biochem J 1994;297:389-397.

15 Gong H, Yan Y, Fang B, Xue Y, Yin P, Li L, Zhang G, Sun X, Chen Z, Ma H, Yang C, Ding Y, Yong Y, Zhu Y, Yang H, Komuro I, Ge J, Zou Y: Knockdown of nucleosome assembly protein 1-like 1 induces mesoderm formation and cardiomyogenesis via notch signaling in murine-induced pluripotent stem cells. Stem Cells 2014;32:1759-1773.

16 Nagata T, Takahashia Y, Ishii Y, Asai S, Nishida Y, Murata A, Koshinaga T, Fukuzawa M, Hamazaki M, Asami K, Ito E, Ikeda H, Takamatsu H, Koike K, Kikuta A, Kuroiwa M, Watanabe A, Kosaka Y, Fujita H, Miyake M, Mugishima H: Transcriptional profiling in hepatoblastornas using high-density oligonucleotide DNA array. Cancer Genet Cytogen 2003;145:152-160.

17 Kidd M, Modlin IM, Mane SM, Camp RL, Eick G, Latich I: The role of genetic markers-nap1l1, mage-d2, and mta1 - in defining small-intestinal carcinoid neoplasia. Ann Surg Oncol 2006;13:253-262. 


\section{Cellular Physiology Cell Physiol Biochem 2016;38:340-350

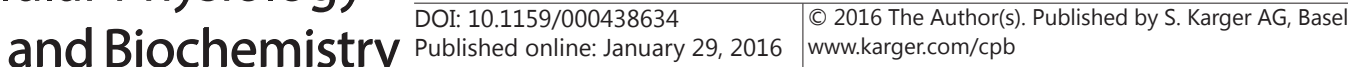

Yan et al.: The Effect of Nap1l1 on the Proliferation of iPSC

18 Pu J, Yuan A, Shan P, Gao E, Wang X, Wang Y, Lau WB, Koch W, Ma XL, He B: Cardiomyocyte-expressed farnesoid-X-receptor is a novel apoptosis mediator and contributes to myocardial ischaemia/reperfusion injury. Eur Heart J 2013;34:1834-1845.

19 Shan P, Pu J, Yuan A, Shen L, Shen L, Chai D, He B: Rxr agonists inhibit oxidative stress-induced apoptosis in h9c2 rat ventricular cells. Biochem Biophys Res Commun 2008;375:628-633.

20 Christensen DR, Calder PC, Houghton FD: Effect of oxygen tension on the amino acid utilisation of human embryonic stem cells. Cell Physiol Biochem 2014;33:237-246.

21 Kellogg DR, Kikuchi A, Fujiinakata T, Turck CW, Murray AW: Members of the nap/set family of proteins interact specifically with b-type cyclins. J Cell Biol 1995;130:661-673.

22 Kellogg DR, Murray AW: Nap1 acts with clb2 to perform mitotic functions and to suppress polar bud growth in budding yeast. J Cell Biol 1995;130:675-685.

23 Altman R, Kellogg D: Control of mitotic events by nap1 and the gin4 kinase. J Cell Biol 1997;138:119-130.

24 Li MF, Strand D, Krehan A, Pyerin W, Heid H, Neumann B, Mechler BM: Casein kinase 2 binds and phosphorylates the nucleosome assembly protein-1 (nap1) in drosophila melanogaster. J Mol Biol 1999;293:1067-1084.

25 Du J, Wu Y, Liu Y, Ai Z, Wu H, Shi X, Guo Z, Zhang Y: E-cadherin is critical for sc1-induced colony growth of f9 embryonic carcinoma cells. Cell Physiol Biochem 2014;33:501-512.

26 Su CC: Tanshinone iia inhibits gastric carcinoma ags cells through increasing p-p38, p-jnk and p53 but reducing p-erk, cdc2 and cyclin b1 expression. Anticancer Res 2014;34:7097-7110.

27 Favata MF, Horiuchi KY, Manos EJ, Daulerio AJ, Stradley DA, Feeser WS, Van Dyk DE, Pitts WJ, Earl RA, Hobbs F, Copeland RA, Magolda RL, Scherle PA, Trzaskos JM: Identification of a novel inhibitor of mitogenactivated protein kinase kinase. J Biol Chem 1998;273:18623-18632.

28 Cheng CW, Chen PM, Hsieh YH, Weng CC, Chang CW, Yao CC, Hu LY, Wu PE, Shen CY: Foxo3a-mediated overexpression of microrna-622 suppresses tumor metastasis by repressing hypoxia-inducible factor1alpha in erk-responsive of lung cancer. Oncotarget DOI:10.18632/oncotarget.

29 Ito T, Bulger M, Kobayashi R, Kadonaga JT: Drosophila nap-1 is a core histone chaperone that functions in atp-facilitated assembly of regularly spaced nucleosomal arrays. Mol Cell Biol 1996;16:3112-3124.

30 Okuwaki M, Kato K, Nagata K: Functional characterization of human nucleosome assembly protein 1-like proteins as histone chaperones. Genes Cells 2010;15:13-27.

31 Simon HU, Mills GB, Kozlowski M, Hogg D, Branch D, Ishimi Y, Siminovitch KA: Molecular characterization of hnrp, a cdna encoding a human nucleosome-assembly-protein-i-related gene product involved in the induction of cell proliferation. Biochem J 1994;297:389-397.

32 Schimmack S, Taylor A, Lawrence B, Alaimo D, Schmitz-Winnenthal H, Buchler MW, Modlin IM, Kidd M: A mechanistic role for the chromatin modulator, nap1l1, in pancreatic neuroendocrine neoplasm proliferation and metastases. Epigenetics Chromatin 2014;7:15.

33 Murray AW: Recycling the cell cycle: Cyclins revisited. Cell 2004;116:221-234.

34 Park HJ, Lyons JC, Ohtsubo T, Song CW: Cell cycle progression and apoptosis after irradiation in an acidic environment. Cell Death Differ 2000;7:729-738.

35 Hsu JD, Kao SH, Ou TT, Chen YJ, Li YJ, Wang CJ: Gallic acid induces g2/m phase arrest of breast cancer cell mcf-7 through stabilization of p27(kip1) attributed to disruption of p27(kip1)/skp2 complex. J Agric Food Chem 2011;59:1996-2003.

36 Xiong Y, Hannon GJ, Zhang H, Casso D, Kobayashi R, Beach D: P21 is a universal inhibitor of cyclin kinases. Nature 1993;366:701-704.

37 Bunz F, Dutriaux A, Lengauer C, Waldman T, Zhou S, Brown JP, Sedivy JM, Kinzler KW, Vogelstein B: Requirement for p53 and p21 to sustain g(2) arrest after DNA damage. Science 1998;282:1497-1501.

38 Zhang J, Wilson GF, Soerens AG, Koonce CH, Yu J, Palecek SP, Thomson JA, Kamp TJ: Functional cardiomyocytes derived from human induced pluripotent stem cells. Circ Res 2009;104:e30-41.

39 Huang WR, Chiu HC, Liao TL, Chuang KP, Shih WL, Liu HJ: Avian reovirus protein p17 functions as a nucleoporin tpr suppressor leading to activation of p53, p21 and pten and inactivation of pi3k/akt/mtor and erk signaling pathways. PloS One 2015;10:e0133699.

40 Chang CH, Li JR, Shu KH, Fu YC, Wu MJ: Hydronephrotic urine in the obstructed kidney promotes urothelial carcinoma cell proliferation, migration, invasion through the activation of mtorc2-akt and erk signaling pathways. PloS One 2013;8:e74300. 